\title{
CYMIAZOLE, A SYSTEMIC ACARICIDE THAT CONTROLS ACARAPIS WOODI (RENNIE) INFESTING HONEY BEES. I. LABORATORY TESTS ${ }^{1}$
}

\author{
Frank A. EISCHEN ', Carlos VERGARA ', Alfred DIETZ ', \\ and David CARDOSO-TAMEZ ${ }^{2}$ \\ 1. Department of Entomology University of Georgia, Athens, Georgia 39602 USA \\ 2. Apicultura Cardoso Apdo. Postal $N^{o} .1$ Buenavista Allende, Nuevo Leon, Mexico
}

\begin{abstract}
Cymiazole (2-[2, 4-dimenthylphenyl-imino]-3-methyl-4-thiazoline-hydrochloride, Apitol ${ }^{\circledR}, \quad 17,5 \%$ cymiazole granules, Ciba-Geigy Ltd.) was dissolved in $50 \%$ sucrose syrup and fed to caged honey bees infested with Acarapis woodi (Rennie). Concentrations of $0.88 \mathrm{mg} / \mathrm{ml}$ and higher killed about $80 \%$ of adult mites within one week. Lower concentrations took longer. After three weeks the $\mathbf{L C}_{50}$ was 0.03 $\mathrm{mg} / \mathrm{ml}$. A similar experiment involving cymiazole mixed in queen cage candy gave a comparable level of control $\left(L C_{50}=0.15 \mathrm{mg} / \mathrm{g}\right)$. No adverse effects were observed in bees given effective doses of cymiazole. Lowered comsumption and shortened longevity were observed when concentrations 3-4 times that which gave adequate control were fed.
\end{abstract}

\section{INTRODUCTION}

For reasons that are not clear, Acarapis woodi has spread rapidly throughout Mexico, the United States, and recently localized infestations have been reported in Canada (Guzman-Novoa and Zozaya-Rubio, 1984 ; DelfinadoBAKER, 1985 ; Anon. 1986b). In addition to becoming widespread, the prevalence of infested honey bee colonies (Apis mellifera) has increased greatly in some regions, especially Mexico (Anon. 1984 ; Guzman-Novoa and ZozaYARuBIo, 1984). Equally important is the high proportion of these colonies that have $>30 \%$ of their bees parasitized (EISCHEN et al. 1988 ; EISCHEN et al., unpublished). The economic consequences of infestations at this level are substantial (Bailey, 1961 ; Guzman-Novoa and Zozaya-Rubio, 1984 ; EIsCHEN, 1987 ; EISCHEN et al., 1989).

Because registered therapeutic treatments are not currently available, legislation was enacted to provide protection for uninfested areas (eg., Anon. 1986a,c,d). However, the large numbers of colonies involved in a highly 
mobile industry make regulation difficult. Clearly, safe medication would help solve this situation. Recently cymiazole (Apitol ${ }^{\circledR}$, Ciba-Geigy), a systemic acaricide has showed promising results in controlling Varroa jacobsoni (RITTER, 1985 ; SCHMID, 1985). Though phylogenetically different, both $V$. jacobsoni and $A$. woodi utilize honey bee hemolymph for food. This suggests the possibility of similar physiology and response to an acaricide.

This report details laboratory experiments designed to evaluate the efficacy of cymiazole for controlling $A$. woodi infesting worker honey bees.

\section{MATERIALS AND METHODS}

Tests were conducted during the summer of 1986 in the facilities of Apicultura Cardoso, which is located in Buenavista Allende, Nuevo Leon, Mexico (lat. $100^{\circ} 01^{\prime}$ north, long. $25^{\circ} 17^{\prime}$ west). Bees of mixed Italian stock (Apis mellifera ligustica Spin.) were obtained locally from colonies heavily infested (70-100\%) with $A$. woodi. Severely infested bees were selected for testing as they are likely to be stressed by the parasite load. If medication exerts an additional stress then it is most easily detected in these bees. This serves to set the upper limits on safe dosages. Secondly, heavy parasite loads present a worst case. If medication is efficacious, then it is also likely to be so at lower levels.

All trials were conducted in laboratory test cages fitted with a small piece of comb (KuLINČEvić and Rothenbuhler, 1973). Thirty or 35 bees were placed in each cage, and one cage was set up for each treatment (i.e. concentration). Mite-infested bees were obtained by removing worker bees from the inner surface of the hive cover of a heavily infested colony. Young uninfested bees were obtained by placing combs of emerging adults in an incubator and collecting bees 24 hours later. Treatments consisted of $50 \%$ sucrose syrup (vol./vol.) or solutions of cymiazole (2-[2, 4-dimethylphenyl-imino]-3-methyl-4thiazoline-hydrochloride) in $50 \%$ sucrose syrup. The commercial preparation of cymiazole is stable in syrup and honey (W.J. Schmid, pers. commun.) Treatments and distilled water were provided ad libitum by means of inverted vials, which had small holes in their caps. All tests were conducted in a darkened incubator $\left(32 \pm 1{ }^{\circ} \mathrm{C}, 80 \pm 5 \% \mathrm{RH}\right)$. Cages were examined daily and the dead bees removed and counted.

\section{Experiment 1. Young Bee Longevity}

Thirty uninfested worker bees (0-24 hrs.) were placed in a laboratory test cage for each treatment (RINDERER and BAXter, 1978). Treatments consisted of $0.0,0.05,0.11,0.22,0.44,0.88,1.75,3.5$, and $7.0 \mathrm{mg} / \mathrm{ml}$ of cymiazole in $50 \%$ sucrose syrup $(12 \mathrm{ml})$. After 14 days all cages received fresh sucrose syrup and distilled water only. Observations were stopped after 15 bees/cage died, i.e. $50 \%$ of the bees (LT 50 .

\section{Experiment 2. Old Bee Longevity}

Thirty bees from a heavily infested colony (70\%) were placed in a laboratory test cage for each treatment. Treatments consisted of $0.0,0.11,0.22,0.44,0.88,1.75,3.5$, and $7.0 \mathrm{mg} / \mathrm{ml}$ of cymiazole in $50 \%$ sucrose syrup $(12 \mathrm{ml})$. Fresh syrups and distilled water were given on days 0,7 , and 14 . An estimate of syrup hoarding was made by counting the number of comb cells containing syrup on the 14th day of treatment. Observations were stopped after 15 bees/cage had died $\left(\mathrm{LT}_{50}\right)$.

\section{Experiment $3 . \mathrm{LC}_{50}$ for $A$. woodi ; Cymiazole in syrup.}

Thirty-five bees from a heavily infested colony (100\%) were placed in a laboratory test cage for each treatment. Treatments consisted of $0.0,0.025,0.05,0.11,0.22,0.44,0.88$, and $1.75 \mathrm{mg} / \mathrm{ml}$ of 
cymiazole in $50 \%$ sucrose syrup $(12 \mathrm{ml})$. Fresh syrups and distilled water were given on days 0,7 , and 14. Five bees/treatment were examined on days 7,14 , and 21 . Dissections and mortality determinations of $A$. woodi were made according to Eischen et al. (1987). Both prothoracic tracheae were inspected and the most heavily infested trachea selected for scoring.

Experiment 4. $\mathrm{LC}_{50}$ for $A$. woodi; Cymiazole in queen cage candy.

Thirty-five bees from a heavily infested colony $(100 \%)$ were placed in a laboratory test cage for each treatment. Treatments consisted of $0.0,0.75,1.0,1.25,1.5,2.0$, and $3.0 \mathrm{mg} / \mathrm{g}$ of cymiazole in candy (confectioners' sugar and $50 \%$ sucrose syrup, $3.7: 1$, see LAIDLAW and ECKERT, 1962). Candy (5.5 g) was pressed into a vial cap and placed on the floor of the test cage. Fresh candies and distilled water were given on days 0 and 7. After 7 days, uneaten candy was weighed. Five bees/treatment were examined on days 7,14 , and 21 .

Data were subjected to probit analysis using a SAS program (RAY, 1982) based on FinNEY (1971). Significant differences were determined by nonoverlap of the $95 \%$ fiducial limits.

\section{RESULTS}

\section{Experiment 1. Young Bee Longevity}

The median life-span of young bees fed $0.05-1.75 \mathrm{mg} / \mathrm{ml}$ cymiazole treated syrups was similar to those fed sucrose syrup $\left(\mathrm{LT}_{50}=21\right.$ days). Bees fed concentrations $3.5 \mathrm{mg} / \mathrm{ml}$ or higher had life-spans about one-half that of controls $\left(\mathrm{LT}_{50}=13\right.$ days $\left.; \mathrm{t}=11.8, \mathrm{P}<0.01\right)$. In general, longevity was rather short even in control bees.

\section{Experiment 2. Old Bee Longevity}

The median life-span of bees fed, $0,0.05,0.11,0.22,0.44,0.88,1.75,3.5$, and $7.0 \mathrm{mg} / \mathrm{ml}$ of cymiazole in syrup was $33,32,21,23,27,27,14,6$, and 3 days, respectively. It is not clear why longevity was relatively short for bees fed the 0.11 and $0.22 \mathrm{mg} / \mathrm{ml}$ syrups. Bees fed cymiazole in concentrations $>$ $0.88 \mathrm{mg} / \mathrm{ml}$ died significantly sooner than controls $(\mathrm{t}=5.62, \mathrm{P}<0.01)$. The shorter life-spans observed at high concentrations may not have been caused totally by toxicity but also involved decreased palatability.

The estimate of hoarding revealed that bees treated with $0,0.025,0.05$, $0.11,0.22,0.44,0.88$, and $1.75 \mathrm{mg} / \mathrm{ml}$ cymiazole had stored $50,58,77,40,42$, 31,43 , and 0 cells of syrup, respectively.

\section{Experiment 3. $L C_{50}$ for A. woodi ; Cymiazole in syrup}

Concentrations of $0.88 \mathrm{mg} / \mathrm{ml}$ or higher were effective after seven days (ca. $80 \%$ adult mite mortality, Table 1). Lower concentrations, eg. $0.05 \mathrm{mg}$ / $\mathrm{ml}$, were not effective until the third week. The lowest concentration $(0.025$ $\mathrm{mg} / \mathrm{ml}$ ) appeared ineffective. The $\mathrm{LC}_{50}$ after three weeks was $0.03 \mathrm{mg} / \mathrm{ml}$, 
TABL. 1. - Survival of Acarapis woodi infesting honey bees fed cymiazole in syrup ${ }^{1}$

\begin{tabular}{|c|c|c|c|c|c|c|}
\hline \multirow[b]{2}{*}{ Treatment } & \multirow[b]{2}{*}{ Day } & \multicolumn{2}{|c|}{ Adults } & \multicolumn{2}{|c|}{ Larvae } & \multirow{2}{*}{$\begin{array}{c}\text { Eggs } \\
(\overline{\mathrm{X}} \pm \mathrm{SE})\end{array}$} \\
\hline & & $\begin{array}{c}\text { Alive } \\
(\overline{\mathrm{X}} \pm \mathrm{SE})\end{array}$ & $\begin{array}{c}\text { Dead } \\
(\overline{\mathrm{X}} \pm \mathrm{SE})\end{array}$ & $\begin{array}{c}\text { Alive } \\
(\overline{\mathrm{X}}+\mathrm{SE})\end{array}$ & $\frac{\text { Dead }}{(\bar{X}+S E)}$ & \\
\hline $\begin{array}{l}\text { Sucrose } \\
\text { control }\end{array}$ & $\begin{array}{r}7 \\
14 \\
21\end{array}$ & $\begin{array}{r}16.2 \pm 1.5 \\
18.2 \pm 1.4 \\
9.8 \pm 2.7\end{array}$ & $\begin{array}{l}4.4 \pm 0.7 \\
3.6 \pm 0.7 \\
4.6 \pm 0.6\end{array}$ & $\begin{array}{l}8.2 \pm 2.4 \\
7.6 \pm 2.7 \\
3.4 \pm 1.0\end{array}$ & $\begin{array}{c}0.0 \\
0.2 \pm 0.2 \\
0.4 \pm 0.2\end{array}$ & $\begin{array}{l}8.8 \pm 1.5 \\
6.2 \pm 2.6 \\
5.4 \pm 3.1\end{array}$ \\
\hline $0.025 \mathrm{mg} / \mathrm{ml}$ & $\begin{array}{r}7 \\
14 \\
21\end{array}$ & $\begin{array}{l}17.4 \pm 2.1 \\
21.2 \pm 4.4 \\
21.4 \pm 6.7\end{array}$ & $\begin{array}{l}5.8 \pm 0.9 \\
7.2 \pm 1.5 \\
6.6 \pm 1.9\end{array}$ & $\begin{array}{l}6.8 \pm 2.3 \\
8.8 \pm 3.1 \\
7.8 \pm 1.0\end{array}$ & $\begin{array}{c}0.0 \\
0.0 \\
0.6 \pm 0.4\end{array}$ & $\begin{array}{l}6.2 \pm 3.3 \\
8.2 \pm 2.2 \\
6.2 \pm 0.9\end{array}$ \\
\hline $0.05 \mathrm{mg} / \mathrm{ml}$ & $\begin{array}{r}7 \\
14 \\
21\end{array}$ & $\begin{array}{l}22.2 \pm 4.5 \\
32.2 \pm 4.2 \\
16.8 \pm 3.1\end{array}$ & $\begin{array}{r}8.2 \pm 2.1 \\
10.6 \pm 1.3 \\
40.4 \pm 6.2\end{array}$ & $\begin{array}{r}17.8 \pm 0.4 \\
20.8 \pm 3.6 \\
7.8 \pm 3.3\end{array}$ & $\begin{array}{c}0.0 \\
0.4 \pm 0.2 \\
0.8 \pm 0.5\end{array}$ & $\begin{array}{r}10.0 \pm 3.1 \\
16.2 \pm 2.3 \\
2.8 \pm 0.8\end{array}$ \\
\hline $0.11 \mathrm{mg} / \mathrm{ml}$ & $\begin{array}{r}7 \\
14 \\
21\end{array}$ & $\begin{array}{l}12.8 \pm 2.7 \\
10.6 \pm 2.3 \\
13.0 \pm 5.4\end{array}$ & $\begin{array}{r}5.4 \pm 1.0 \\
10.8 \pm 1.7 \\
26.4 \pm 3.8\end{array}$ & $\begin{array}{l}8.0 \pm 1.6 \\
6.0 \pm 1.0 \\
4.4 \pm 1.5\end{array}$ & $\begin{array}{l}0.4 \pm 0.2 \\
0.4 \pm 0.4 \\
0.8 \pm 0.4\end{array}$ & $\begin{array}{l}5.4 \pm 2.3 \\
8.0 \pm 1.1 \\
3.2 \pm 1.2\end{array}$ \\
\hline $0.22 \mathrm{mg} / \mathrm{ml}$ & $\begin{array}{r}7 \\
14 \\
21\end{array}$ & $\begin{array}{r}17.0 \pm 4.2 \\
14.6 \pm 4.9 \\
7.6 \pm 2.1\end{array}$ & $\begin{array}{r}6.6 \pm 2.2 \\
9.6 \pm 2.9 \\
33.6 \pm 6.0\end{array}$ & $\begin{array}{r}10.6 \pm 2.2 \\
3.8 \pm 2.0 \\
4.2 \pm 1.0\end{array}$ & $\begin{array}{c}0.0 \\
2.0 \pm 1.0 \\
0.2 \pm 0.2\end{array}$ & $\begin{array}{l}6.0 \pm 2.3 \\
3.4 \pm 2.0 \\
2.4 \pm 0.7\end{array}$ \\
\hline $0.44 \mathrm{mg} / \mathrm{ml}$ & $\begin{array}{r}7 \\
14 \\
21\end{array}$ & $\begin{array}{r}11.4 \pm 2.0 \\
8.2 \pm 3.8 \\
8.4 \pm 3.2\end{array}$ & $\begin{array}{r}6.4 \pm 1.4 \\
11.2 \pm 3.2 \\
24.4 \pm 4.8\end{array}$ & $\begin{array}{l}3.2 \pm 0.9 \\
1.2 \pm 0.6 \\
1.4 \pm 0.7\end{array}$ & $\begin{array}{l}0.2 \pm 0.2 \\
0.4 \pm 0.4 \\
0.6 \pm 0.4\end{array}$ & $\begin{array}{c}2.8 \pm 1.7 \\
0.0 \\
1.0 \pm 0.5\end{array}$ \\
\hline $0.88 \mathrm{mg} / \mathrm{ml}$ & $\begin{array}{r}7 \\
14 \\
21\end{array}$ & $\begin{array}{l}3.2 \pm 0.7 \\
4.8 \pm 1.6 \\
1.6 \pm 0.5\end{array}$ & $\begin{array}{l}15.8 \pm 3.5 \\
13.4 \pm 2.6 \\
16.4 \pm 5.0\end{array}$ & $\begin{array}{c}2.2 \pm 0.7 \\
0.2 \pm 0.2 \\
0.0\end{array}$ & $\begin{array}{c}3.2 \pm 0.7 \\
0.0 \\
0.4 \pm 0.4\end{array}$ & $\begin{array}{l}1.4 \pm 0.6 \\
1.0 \pm 0.3 \\
0.4 \pm 0.4\end{array}$ \\
\hline $1.75 \mathrm{mg} / \mathrm{ml}$ & $\begin{array}{r}7 \\
14 \\
21\end{array}$ & $\begin{array}{l}1.8 \pm 1.6 \\
6.0 \pm 2.3 \\
1.6 \pm 0.5\end{array}$ & $\begin{array}{l}16.0 \pm 2.8 \\
11.4 \pm 3.2 \\
18.2 \pm 1.2\end{array}$ & $\begin{array}{c}4.4 \pm 0.9 \\
0.2 \pm 0.2 \\
0.0\end{array}$ & $\begin{array}{c}0.0 \\
0.4 \pm 0.2 \\
0.2 \pm 0.2\end{array}$ & $\begin{array}{l}0.2 \pm 0.2 \\
0.8 \pm 0.6 \\
1.6 \pm 0.2\end{array}$ \\
\hline
\end{tabular}

1. Values are mites/trachea $(\mathrm{N}=5$ bees).

TABL. 2. - Response of adult Acarapis woodi infesting honey bees to cymiazole

\begin{tabular}{lc|c|c|c}
\hline \hline \multicolumn{2}{c|}{ Day evaluated } & $\mathrm{LC}_{50}(\mathrm{mg} / \mathrm{ml})$ & $95 \%$ Fiducial Limits & Slope $\pm \mathrm{SE}$ \\
\hline \multirow{2}{*}{ Syrup } & & & & \\
& 7 & 0.29 & $0.09-3.13$ & $8.7 \pm 0.28$ \\
& 14 & 0.27 & $0.14-0.77$ & $5.3 \pm 0.14$ \\
& 21 & 0.03 & $0.007-0.09$ & $7.2 \pm 0.29$ \\
\multirow{2}{*}{ Candy } & & & & \\
& 21 & 0.15 & $0.005-0.36$ & $14.2 \pm 0.42$ \\
\hline
\end{tabular}

1. $\mathrm{LC}_{50}$ for syrup and candy expressed in $\mathrm{mg} / \mathrm{ml}$ and $\mathrm{mg} / \mathrm{g}$, respectively. 
which was significantly smaller than evaluations made after 7 and 14 days $(P=0.05$, Table 2$)$. Additional information was gained by examining mite survival. By the third week, egg production had dropped in all treatments with the exception of the lowest concentration $(0.025 \mathrm{mg} / \mathrm{ml})$ and control (Table 1). Similar data for larvae were observed (Table 1). Reduced larval numbers in the higher concentrations may be caused by reduced egg production as well as mortality, though appreciable larval mortality was observed only at the $0.88 \mathrm{mg} / \mathrm{ml}$ concentration (59\%, Table 1$)$.

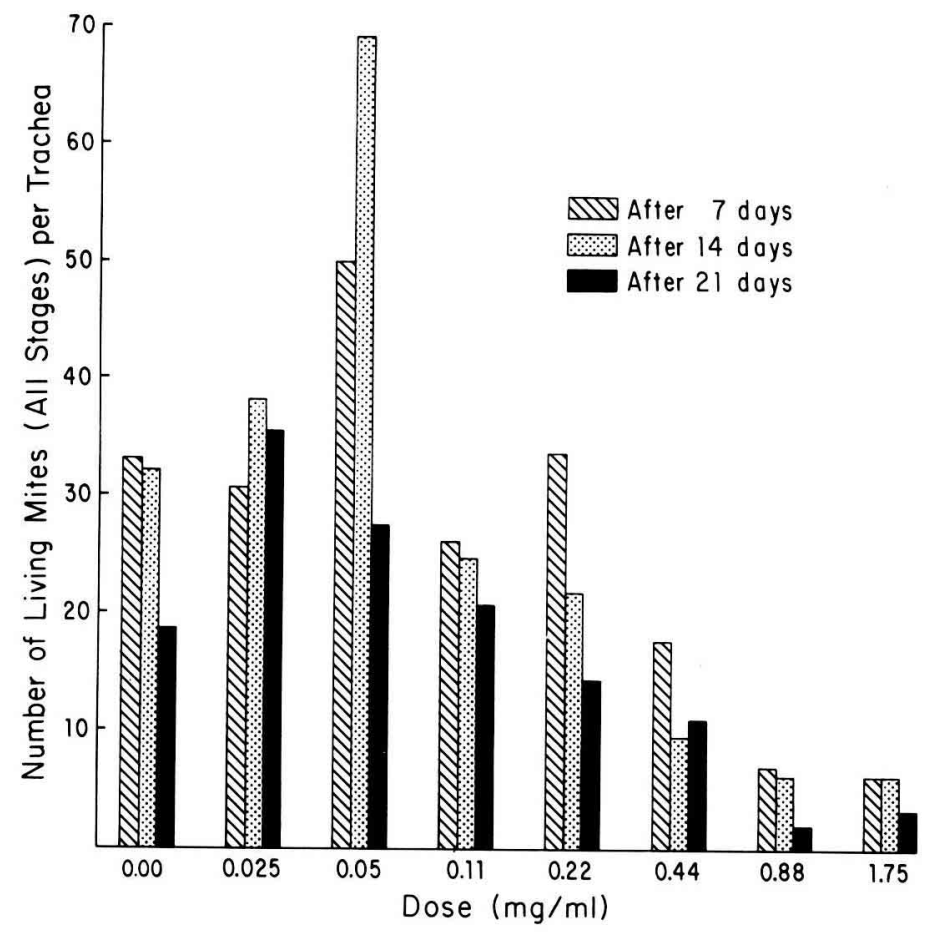

Fig. 1. - Survival of Acarapis woodi (all stages) infesting honey bees fed cymiazole in syrup.

A composite of all surviving life stages is shown in Fig. 1. A decrease in the number of living mites is evident as a factor of both increasing concentration and time. After three weeks, $18.6 \mathrm{mites} /$ trachea were observed in the controls, while less than two living mites/trachea were observed at the 0.88 and $1.75 \mathrm{mg} / \mathrm{ml}$ concentrations (Table 1). The latter is likely to be below the economic threshold (EISCHEN, 1987 ; EISCHEN et al., 1989). Bee mortality after 32 days was $0,8.6,0,0,14.3,100,20,100$, and $100 \%$ when fed syrups containing $0.0,0.025,0.05,0.11,0.22,0.44,0.88,1.75$, and $3.5 \mathrm{mg} / \mathrm{ml}$ of cymiazole, respectively. 
TABL. 3. - Survival of Acarapis woodi infesting honey bees fed cymiazole in queen cage candy ${ }^{1}$

\begin{tabular}{|c|c|c|c|c|c|c|}
\hline \multirow[b]{2}{*}{ Treatment } & \multirow[b]{2}{*}{ Day } & \multicolumn{2}{|c|}{ Adults } & \multicolumn{2}{|c|}{ Larvae } & \multirow{2}{*}{$\begin{array}{c}\text { Eggs } \\
(\overline{\mathrm{X}} \pm \mathrm{SE})\end{array}$} \\
\hline & & $\begin{array}{c}\text { Alive } \\
(\overline{\mathrm{X}} \pm \mathrm{SE})\end{array}$ & $\begin{array}{c}\text { Dead } \\
(\overline{\mathrm{X}} \pm \mathrm{SE})\end{array}$ & $\begin{array}{c}\text { Alive } \\
(\overline{\mathrm{X}}+\mathrm{SE})\end{array}$ & $\begin{array}{c}\text { Dead } \\
(\overline{\mathrm{X}}+\mathrm{SE})\end{array}$ & \\
\hline $\begin{array}{l}\text { Sucrose } \\
\text { control }\end{array}$ & $\begin{array}{r}7 \\
14 \\
21\end{array}$ & $\begin{array}{l}33.0 \pm 7.0 \\
25.2 \pm 3.0 \\
23.4 \pm 1.3\end{array}$ & $\begin{array}{r}4.8 \pm 0.4 \\
14.2 \pm 2.8 \\
9.6 \pm 2.7\end{array}$ & $\begin{array}{r}3.4 \pm 1.3 \\
10.4 \pm 3.4 \\
9.2 \pm 1.8\end{array}$ & $\begin{array}{c}0.0 \\
0.0 \\
0.6 \pm 0.2\end{array}$ & $\begin{array}{l}3.6 \pm 2.2 \\
8.8 \pm 2.4 \\
4.4 \pm 1.0\end{array}$ \\
\hline $0.75 \mathrm{mg} / \mathrm{g}$ & $\begin{array}{r}7 \\
14 \\
21\end{array}$ & $\begin{array}{r}22.2 \pm 4.2 \\
6.6 \pm 1.9 \\
4.8 \pm 1.1\end{array}$ & $\begin{array}{r}5.6 \pm 1.4 \\
10.4 \pm 3.1 \\
17.4 \pm 3.2\end{array}$ & $\begin{array}{l}4.6 \pm 1.4 \\
1.6 \pm 0.8 \\
2.4 \pm 0.6\end{array}$ & $\begin{array}{c}0.0 \\
2.0 \pm 0.7 \\
1.4 \pm 0.2\end{array}$ & $\begin{array}{l}0.4 \pm 0.4 \\
1.2 \pm 0.8 \\
2.2 \pm 0.7\end{array}$ \\
\hline $1.0 \mathrm{mg} / \mathrm{g}$ & $\begin{array}{r}7 \\
14 \\
21\end{array}$ & $\begin{array}{r}16.6 \pm 1.6 \\
9.8 \pm 2.6 \\
2.0 \pm 1.0\end{array}$ & $\begin{array}{r}5.0 \pm 0.4 \\
15.8 \pm 2.6 \\
13.4 \pm 1.8\end{array}$ & $\begin{array}{l}1.2 \pm 0.4 \\
0.4 \pm 0.4 \\
0.2 \pm 0.2\end{array}$ & $\begin{array}{c}0.0 \\
0.4 \pm 0.2 \\
0.6 \pm 0.4\end{array}$ & $\begin{array}{l}1.0 \pm 0.3 \\
0.4 \pm 0.4 \\
0.4 \pm 0.4\end{array}$ \\
\hline $1.25 \mathrm{mg} / \mathrm{g}$ & $\begin{array}{r}7 \\
14 \\
21\end{array}$ & $\begin{array}{r}21.8 \pm 3.8 \\
16.0 \pm 6.1 \\
3.0 \pm 0.5\end{array}$ & $\begin{array}{r}5.0 \pm 1.1 \\
12.6 \pm 2.1 \\
17.6 \pm 2.6\end{array}$ & $\begin{array}{c}3.0 \pm 1.0 \\
0.2 \pm 0.2 \\
0.0\end{array}$ & $\begin{array}{c}0.0 \\
0.2 \pm 0.2 \\
0.2 \pm 0.2\end{array}$ & $\begin{array}{l}2.0 \pm 1.3 \\
0.6 \pm 0.4 \\
1.2 \pm 0.6\end{array}$ \\
\hline $1.5 \mathrm{mg} / \mathrm{g}$ & $\begin{array}{r}7 \\
14 \\
21\end{array}$ & $\begin{array}{r}13.6 \pm 5.3 \\
9.0 \pm 3.7 \\
4.4 \pm 1.5\end{array}$ & $\begin{array}{r}5.6 \pm 1.0 \\
24.0 \pm 3.1 \\
26.2 \pm 7.5\end{array}$ & $\begin{array}{l}2.0 \pm 0.9 \\
0.6 \pm 0.6 \\
2.2 \pm 0.9\end{array}$ & $\begin{array}{c}0.0 \\
0.0 \\
0.4 \pm 0.4\end{array}$ & $\begin{array}{l}1.8 \pm 0.4 \\
0.8 \pm 0.6 \\
1.0 \pm 0.5\end{array}$ \\
\hline $2.0 \mathrm{mg} / \mathrm{g}$ & $\begin{array}{r}7 \\
14 \\
21\end{array}$ & $\begin{array}{r}24.4 \pm 8.7 \\
4.6 \pm 2.6 \\
3.0 \pm 0.5\end{array}$ & $\begin{array}{r}7.8 \pm 1.2 \\
14.4 \pm 3.7 \\
31.4 \pm 1.9\end{array}$ & $\begin{array}{c}1.4 \pm 0.9 \\
0.0 \\
0.2 \pm 0.2\end{array}$ & $\begin{array}{c}0.0 \\
0.4 \pm 0.4 \\
0.6 \pm 0.2\end{array}$ & $\begin{array}{c}0.6 \pm 0.4 \\
0.4 \pm 0.4 \\
0.0\end{array}$ \\
\hline $3.0 \mathrm{mg} / \mathrm{g}$ & $\begin{array}{r}7 \\
14 \\
21\end{array}$ & $\begin{array}{c}8.0 \pm 0.9 \\
11.4 \pm 3.1 \\
\text { high bee m }\end{array}$ & $\begin{array}{r}24.4 \pm 5.0 \\
32.2 \pm 2.9 \\
\text { tality }\end{array}$ & $\begin{array}{l}2.6 \pm 0.7 \\
1.4 \pm 0.6\end{array}$ & $\begin{array}{l}1.2 \pm 0.2 \\
0.4 \pm 0.2\end{array}$ & $\begin{array}{l}2.0 \pm 0.3 \\
0.6 \pm 0.2\end{array}$ \\
\hline
\end{tabular}

1. Values are mites/trachea $(\mathrm{N}=5$ bees $)$.

Experiment 4. $L C_{50}$ for A. woodi ; Cymiazole in queen cage candy.

Initially, only the highest concentration gave control, but after three weeks all concentrations showed activity (Table 3 ). The $\mathrm{LC}_{50}$ for cymiazole after 21 days was $0.15 \mathrm{mg} / \mathrm{g}$ (Table 2). Mite survival (all stages) ranged from 40 mites/ trachea in controls to 2.6 in bees given the $1.0 \mathrm{mg} / \mathrm{g}$ diet (Fig. 2 and Table 3). Candy consumption differed considerably. After one week, bees fed the 0 , $0.75,1.0,1.25,1.5$, and $2.0 \mathrm{mg} / \mathrm{g}$ treatments consumed $98.2,61.8,61.8,56.4$, 52.7 , and $41.8 \%$ of the candy, respectively. This indicates that even the lowest concentration, $0.75 \mathrm{mg} / \mathrm{g}$, reduced palatability. Bee mortality after $2 \mathrm{i}$ days on these same diets was $5.7,0,0,2.8,17.1$, and $22.9 \%$ respectively. 


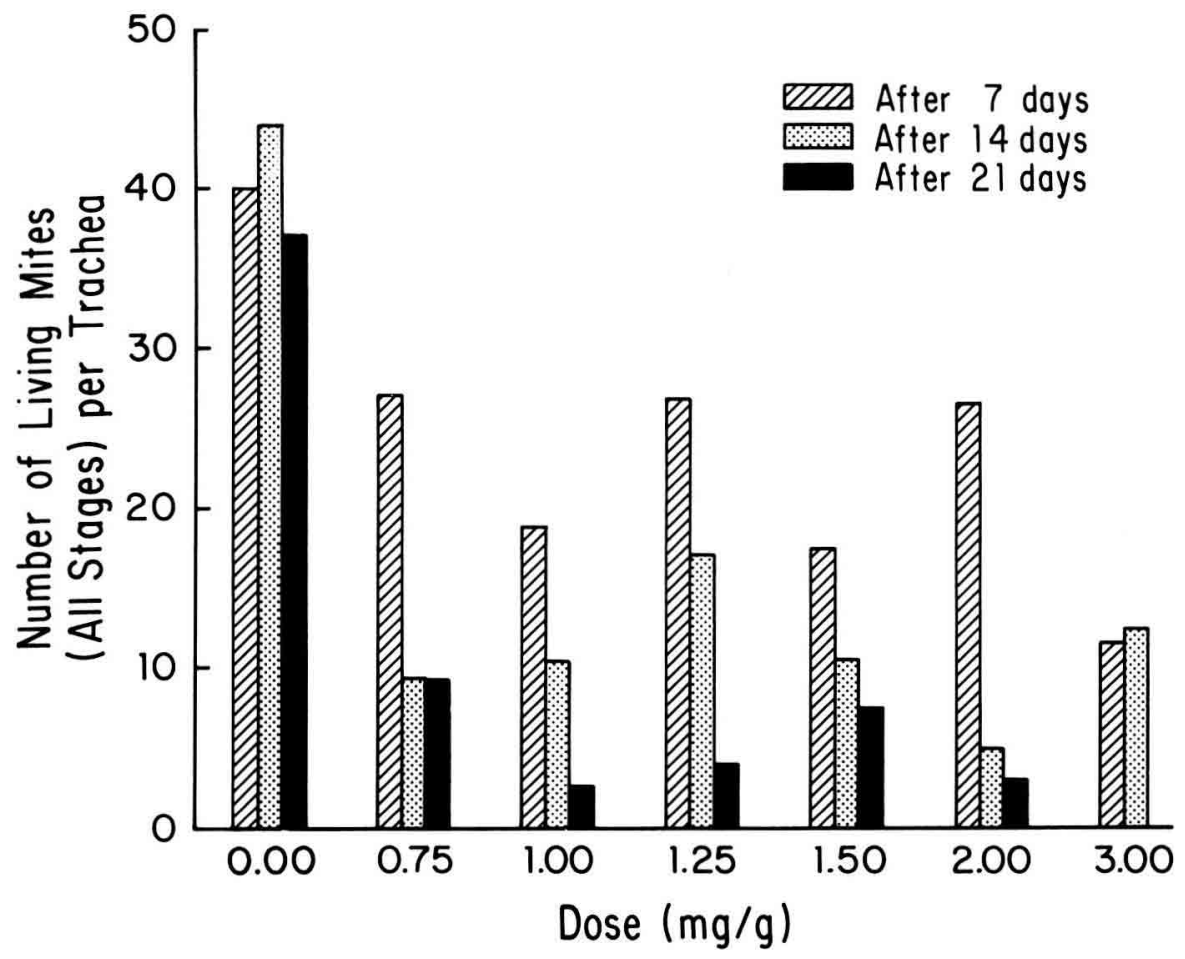

FIG. 2. - Survival of Acarapis woodi (all stages) infesting honey bees fed cymiazole in queen cage candy.

\section{DISCUSSION}

These laboratory tests have shown that, when dissolved in sucrose solutions, cymiazole can control $A$. woodi infesting worker honey bees. Total control was generally not achieved, but $80 \%$ mortality was regularly observed. Mite response to cymiazole was comparatively slow, though this is not likely to be an important factor when treating colonies. The poor control of immature mites is not surprising as they probably feed less than adults (see Morison, 1932, ÖrösI-PÁL, 1934). This has been observed previously with other effective adult acaricides (VeCCHI and GIORDANI, 1968 ; EISCHEN et al., 1987).

Why incomplete adult control was observed is not clear. Lowered palatability at high concentrations may have caused bees to limit the dose by consuming less medicated syrup. Perhaps low chronic doses are worth further examination. Alternatively, these observations were made on caged, queenless, and broodless bees. Consumption rates and physiologies might be quite different under more natural conditions. It is encouraging though, that cymiazole 
was able to effect acceptable control at levels that did not appear to harm the bees.

Received for publication in October 1987. Accepted for publication in June 1988.

\section{ACKNOWLEDGMENTS}

We thank W.G. HART USDA-ARS Subtropical Insects Laboratory, T.E. Rinderer, USDA-ARS Honey Bee Breeding, Genetics \& Physiology Research Laboratory, and H. ShIMANUKI, USDA-ARS Beneficial Insects Laboratory for advice ans assistance while in Mexico. W.T. WILSON, USDA-ARS Honey Bee Laboratory provided assistance while in Mexico, as well as critiquing the manuscript. G.O. WARE heiped with the statistical analysis. Our special thanks go to W.J. ScHmid, Ciba-Geigy Switzerland, who arranged for support through Research Grant 25-21-RC293-108.

\section{RÉSUMÉ}

\section{LE CYMIAZOLE, UN ACARICIDE SYSTÉMIQUE CONTRE ACARAPIS WOODI (RENNIE), PARASITE DE L'ABEILLE . I. TESTS EN LABORATOIRE}

Le cymaziole (hydrochlorure de 2-[2, 4-dimethylphenyl-imino]-3-methyl-4-thiazoline; spécialité Apitol ${ }^{\circledR}$ de Ciba-Geigy Ltd. sous forme de granules dosés à $17,5 \%$ de cymiazole) a été dissout dans un sirop de saccharose à $50 \%$ (vol./vol.) et donné à des abeilles encagées, parasitées par Acarapis woodi. Les sirops contenaient $0,0,0,025,0,05,0,11,0,22,0,44,0,88$ et $1,75 \mathrm{mg}$ de cymiazole par $\mathrm{ml}$. Les deux plus fortes concentrations ont tué environ $80 \%$ des acariens adultes en une semaine. Au bout de 3 semaines on a observé des résultats plutôt bons dans tous les groupes traités, excepté chez ceux qui avaient reçu le sirop à $0,025 \mathrm{mg}$ de cymiazole par $\mathrm{ml}$. La $\mathrm{CL}_{50}$ à 3 semaines a été de $0,03 \mathrm{mg} / \mathrm{ml}$. Les abeilles nourries avec les sirops à $0,025-0,88 \mathrm{mg}$ de cymiazole par $\mathrm{ml}$ ont eu un comportement d'amassement et une longévité à peu près semblables à ceux des témoins (sirop de sucre pur). Aux concentrations plus élevées, on a observé une consommation plus faible et une longévité réduite. Dans une expérience semblable avec du cymiazole mélangé à du candi dans une cage à reine, on a obtenu un niveau comparable d'efficacité. ( $\mathrm{CL}=0,15 \mathrm{mg} / \mathrm{g}$ ).

\section{ZUSAMMENFASSUNG}

\section{CYMIAZOL (APITOL ${ }^{\circledR}$ ), EIN SYSTEMISCHES AKARAZID ZUR BEKÄMPFUNG VON ACARAPIS WOODI (RENNIE) BEI DER HONIGBIENE. I. LABORTESTS}

Cymiazol (2 - [2,4 - Dimethylphenyl - iminol - 3 - methyl - 4 - thiazolin - hydrochloride, Apitol ${ }^{\circledR}$, $17.5 \%$ Cymiazol Granulat, Ciba-Geigy Ltd.) wurde in $50 \%$ igem Saccharosesirup (Vol./Vol.) gelöst und an gekäfigte Honigbienen, die mit Acarapis woodi (Rennie) infiziert waren, verfüttert. Es wurden Sirupe der Konzentrationen $0.0,0.025,0.05,0.11,0.22,0.44,0.88$ und $1.75 \mathrm{mg} / \mathrm{ml}$ verglichen. Die beiden höchsten Konzentrationen töteten innerhalb einer Woche ca. $80 \%$ der adulten Milben. Nach drei Wochen war die Bekämpfung in allen behandelten Gruppen hinreichend gut außer bei der Konzentration $0.025 \mathrm{mg} / \mathrm{ml}$. Die $\mathrm{LC}_{50}$ war nach drei Wochen $0.03 \mathrm{mg} / \mathrm{ml}$. Das Futtereintragen und die Lebensdauer der Bienen, die mit Cymiazol der Konzentrationen $0.025-0.88 \mathrm{mg} / \mathrm{ml}$ gefüttert wurden, waren in etwa gleich mit dem von mit Saccharosesirup gefütterten Bienen. Bei höheren Konzentrationen wurde eine reduzierte Nahrungsaufnahme und verkürzte Lebensdauer der Bienen festgestellt. Ein ähnliches Experiment mit Cymiazol in Futterteig ergab einen vergleichbaren Wirkungsgrad in der Bekämpfung $\left(\mathrm{LC}_{50}=0.15 \mathrm{mg} / \mathrm{g}\right)$. 


\section{REFERENCES}

Anonymous, 1984. - « Rio Grande valley mite survey. " Speedy Bee, 13, 4.

Anonymous, 1986a. - East Canada closed to U.S. Bee Shipments. Speedy Bee, 14 (3), 1.

Anonymous, 1986b. - Manitoba quarantines tracheal mite infested colonies. Am. Bee J., $126,589$.

Anonymous, 1986c. - Texas holds fast on Rio Grande valley quarantine. Speedy Bee, 15 (1), 14.

Anonymous, 1986d. - Tracheal mite control area established in California. Am. Bee J., 126, 782 and 784.

BaILeY L., 1961. - The natural incidence of Acarapis woodi (Rennie) and the winter mortality of honeybee colonies. Bee World, 42, 96-100.

Delfinado-Baker M., 1985, - An acarologist's view: The spread of the tracheal mite of honey bees in the United States. Am. Bee J., 125, 689-690.

Eischen F.A., 1987. - Overwintering performance of honey bee colonies heavily infested with Acarapis woodi (Rennie). Apidologie, 18, 293-303.

Eischen F.A., Pettis J.S. and Dietz A., 1987. - A rapid method of evaluating compounds for the control of Acarapis woodi (Rennie). Am. Bee J., 127, 99-101.

Eischen F.A., Wilson W.T., Hurley D. and Cardoso-Tamez D., 1988. - Cultural practices that reduce populations of Acarapis woodi (Rennie). Am. Bee J., 128, 209-211.

Eischen F.A., Cardoso-Tamez D., Wilson W.T. and Dietz A., 1989. - Honey production of honey bee colonies infested with Acarapis woodi (Rennie). Apidologie, 20 (1) (in press).

Finney D.J., 1971. - Probit analysis, 3rd ed. Cambridge University Press. Cambridge, England.

Guzman-Novoa E. and Zozaya-Rubio A., 1984. - The effects of chemotherapy on the level of infestation and production of honey in colonies of honey bees with acariosis. Am. Bee J., 124, 669672.

KulinčeviĆ Jovan M. and Rothenbuhler W.C., 1973. - Laboratory and field measurements of hoarding behaviour in the honeybee. J. Apic. Res., 12, 179-182.

Laidlaw H.H. Jr. and Eckert J.E., 1962. - Queen Rearing. University of California Press. Berkeley, California.

Morison G.D., 1932. - A mite «Acarapis" that dwells on the back of the honey-bee. The Bee Kingdom. Leaflet $n^{\circ} 16$.

Ö́ösı-PÁl Z., 1934. - Experiments on the feeding habits of the Acarapis mites. Bee World, $15,93-94$.

Ray A.A. (ed.), 1982. - SAS user's guide : basics. SAS Institute, Cary, N.C.

Rinderer T.E. and BAxter J.R., 1978. - Honey Bees: The effect of group size on longevity and hoarding in laboratory cages. Ann. Entomol. Soc. Am., 71, 732.

Rrtrer W., 1985. - First results from biological trials with Apitol, a medicament with systemic activity. Proc. 30th Int. Apic. Congr. Nagoya, Japan.

Schmid W.J., 1985. - Apitol ${ }^{\circledR}$ - A new acaricide with systemic activity against Varroa mites. Proc. 30th Int. Apic. Congr. Nagoya, Japan.

VeCchi M.A and Giordani G., 1968. - Chemotherapy of acarine disease. 1. Laboratory tests. $J$. Invertebr. Pathol., 10, 390-416. 